\title{
Spinal Gunshot Wounds: Pattern and Associated Lesions in Civilians
}

\author{
Jorge Arturo Cabrera Escamilla, Jorge Álvaro González Ross, José Manuel Pérez Atanasio, \\ Gustavo Casas Martínez, Arelhi González Cisneros, Jose Jimenez Avila \\ Instituto Mexicano del Seguro Social, Unidad Médica de Alta Especialidad "Dr. Victorio de la Fuente Narváez", Mexico City, Mexico
}

\begin{abstract}
Study Design: Retrospective, descriptive case series study.
Purpose: To investigate the frequency, bone pattern, and associated lesions to the spine of gunshot wounds.

Overview of Literature: Gunshot wounds are penetrating in nature and are caused by a bullet or projectile. These are becoming more common and are associated with significant sequelae, requiring long and costly multidisciplinary treatment. Associated spinal cord injuries (SCls) in the civilian population represent $13 \%-17 \%$ of all spinal traumas. Spinal gunshot wounds are commonly thought to be stable; however, there is potential acute and chronic spinal instability if the bullet passes transversely, fracturing either both pedicles or both facet joints.

Methods: We obtained data from the clinical files of patients with spinal gunshot wounds treated by spine surgeons. We performed a statistical analysis to obtain the pattern and frequency of the injuries.

Results: We included 54 patients (48 men [89\%] and six women [11\%]). Eight patterns of spinal gunshot wounds were identified; $61 \%$ (33 patients) had complete SCI. The thoracic spine was most frequently affected, with $88 \%$ of patients having SCl; $75 \%$, hemopneumothorax; $28 \%$, pulmonary lesions; and 16\%, hepatic lesions. The presence of pedicle fractures had an odds ratio of 3.64 for SCI. Conclusions: The two main bone patterns are the vertebral body and posterior arch with pedicle fractures as modifiers, given that they are at a high risk for SCI. The spinal pattern and associated lesions were related to the bullet's path, with the vertebral body having the highest frequency of associated lesions. Vertebral body burst fracture was the main indication for instrumentation.
\end{abstract}

Keywords: Bullet wound; Spinal injuries; Civilian; Soft tissue injuries; Spinal cord injuries

\section{Introduction}

Gunshot wounds are penetrating in nature and are caused by a bullet or projectile and by newly formed concurrent elements at the time the shot is fired [1]. Although the incidence of firearm crime has declined in some countries, the situation has worsened in developing countries $[2,3]$.
Gunshot wounds are classified as low-velocity projectiles $(<1,000 \mathrm{ft} / \mathrm{sec}$ or $304 \mathrm{~m} / \mathrm{sec})$ or high-velocity projectiles ( $>2,000 \mathrm{ft} / \mathrm{sec}$ or $600 \mathrm{~m} / \mathrm{sec}$ ), although moderatevelocity gunshot wounds have also been reported $(600 \mathrm{~m} /$ sec) $[1,4,5]$. Low-velocity handgun shells cause less tissue damage and spinal instability than high-power shells $[1,3,6]$.

Received Sep 21, 2017; Revised Nov 25, 2017; Accepted Dec 5, 2017

Corresponding author: Arelhi González Cisneros

Instituto Tecnologico y de Estudios Superiores de Monterrey Campus Guadalajara, Av. General Ramón Corona 2514 Col. Nuevo México, Zapopan, Jalisco 45201, Mexico

Tel: +52-33-3129-0260, E-mail: arelhigonzalezcisneros@gmail.com 
Spinal gunshot wounds are becoming more common and are associated with significant sequelae, requiring long and costly multidisciplinary treatment [1]. More than half of patients with non-fatal gunshot wounds require hospitalization [7]. Associated spinal cord injuries (SCIs) in the civilian population represent $13 \%-17 \%$ of all spinal traumas and are among the most devastating sequelae $[1,4,7,8]$.

Associated injuries to other organs will often be revealed during the initial assessment, and these injuries could have priority over spinal gunshot wounds and SCIs. After evaluating the airway, breathing, and circulation, neurological examination can be performed. Physical examination has a sensitivity of $100 \%$ and a specificity of $87.5 \%$ for SCIs and critical lesions requiring stabilization $[5,8]$.

Neurological deficits have been reported in 33\%-92.4\% of patients. Most spinal gunshot wounds affect the cervical or thoracic spine with a complete SCI. Neurological examination should be repeated hourly in patients with incomplete neurological injuries so that any change in function can be quickly determined and treated [5].

The definitive treatment for a spinal gunshot wound is determined by neurological function, mechanical spinal stability, and associated lesions in other organs. These factors should be evaluated after immediate medical stabilization of the patient $[5,6]$.

Spinal gunshot wounds are commonly thought to be stable. In the lumbar spine, however, there is potential acute and chronic spinal instability if the bullet passes transversely, fracturing either both pedicles or both facet joints $[3,6]$.

Spinal instability is not observed when a pedicle or facet remains intact. Therefore, careful radiographic evaluation is necessary. If the stability is questionable, flexion and extension X-rays or computed tomography (CT) scans might be necessary $[3,8]$. Fractures of the vertebral body and of posterior elements can be quickly evaluated with a CT scan $[5,7,9]$.

Current evidence in the literature comparing the efficacy between conservative and surgical treatment is inconclusive [4]; therefore, the decision to choose either treatment is complex and challenging $[6,10]$. At times, visceral debridement and broad-spectrum antibiotic therapy appear to be sufficient in patients with a stable spine [10]. Antibiotic therapy for 48-72 hours with broad-spectrum antibiotics should be immediately initiated in all cases
[11-14], especially in patients with perforation of the lower gastrointestinal (GI) tract [12].

Surgical indications are limited and include progressive neurological deficit or incomplete cauda equina injury caused by spinal canal occupation, persistence of cerebrospinal fluid fistula, infection, or spinal instability $[5,6]$. The onset or progression of neurological deterioration is an indication for urgent decompression [15]. Otherwise, treatment should remain conservative, regardless of the neurological grade or the number of injured vertebral bodies $[4,6,10,12,14-17]$.

\section{Materials and Methods}

In this retrospective, case series study, we reviewed physical and electronic files of patients with spinal gunshot wounds treated by the spine trauma department between January 2001 and November 2016. We evaluated the CT scan of each patient for bone patterns and instability. We also reviewed the reports of shock unit doctors, intensive care doctors, and general surgeon notes, to determine reported associated lesions. Incomplete files and patients with no CT scan were excluded. We performed a statistical analysis to obtain the pattern and frequency of spinal and associated injuries using Epi Info ver. 7.2.0.1 (Centers for Disease Control and Prevention, Atlanta, GA, USA).

This study was approved as in compliance with the ethical standards of the local research and ethics committee of the Mexican Social Security Institute (CLIS 2016-3401$62)$.

\section{Results}

We included the files of 54 patients, of which 48 (89\%) were men and six $(11 \%)$ were women. The age range was 9-75 years, with a mean of $30 \pm 13$ years. All patients were

Table 1. Distribution of spinal cord injury severity

\begin{tabular}{lc|}
\hline Spinal cord injury severity & Frequency \\
\hline Frankel A & 33 \\
\hline Frankel B & 3 \\
\hline Frankel C & 1 \\
\hline Frankel D & 2 \\
\hline Frankel E & 14 \\
\hline Total & 53 \\
\hline
\end{tabular}


Table 2. Distribution of spinal cord lesion severity by injured segment

\begin{tabular}{|c|c|c|c|c|c|c|}
\hline \multirow{2}{*}{ Spinal segment } & \multirow{2}{*}{ Total frequency } & \multicolumn{5}{|c|}{ Spinal cord injury severity } \\
\hline & & Frankel A & Frankel B & Frankel C & Frankel D & Frankel E \\
\hline Upper cervical spine & 1 & 0 & 0 & 0 & 0 & 1 \\
\hline Subaxial cervical spine & 7 & 3 & 0 & 0 & 1 & 3 \\
\hline Thoracic spine & 32 & 28 & 0 & 0 & 0 & 4 \\
\hline Lumbar spine & 13 & 2 & 3 & 1 & 1 & 6 \\
\hline Total & 53 & 33 & 3 & 1 & 2 & 14 \\
\hline
\end{tabular}

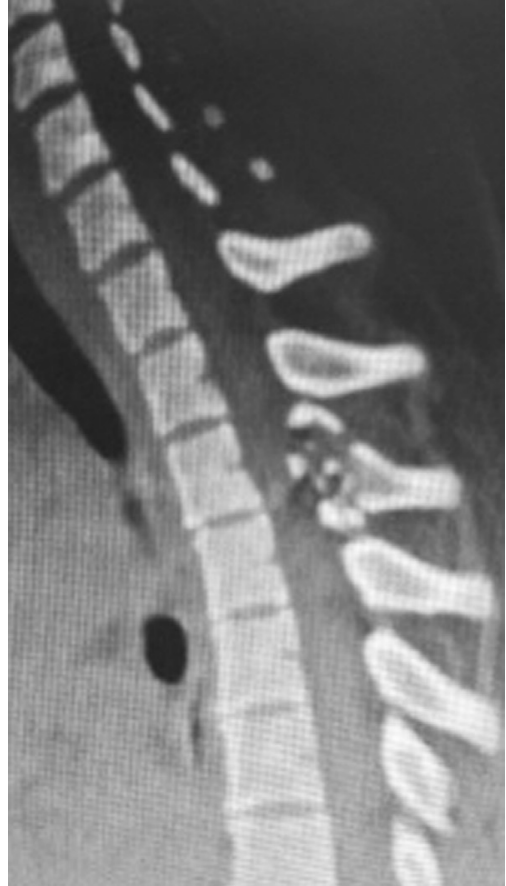

Fig. 1. Posterior arch fracture: sagittal projection with posterior arch fracture.

civilians and had received a low-velocity gunshot wound.

Of the 54 patients, $61 \%$ (33 patients) had complete SCI, $11 \%$ (six patients) had incomplete SCI, and 27\% (15 patients) had no SCI, as shown in Table 1. The greatest spinal cord involvement was observed in the thoracic spine, as shown in Table 2 .

In the CT scan evaluation, we found eight different fracture patterns, shown in Table 3. These patterns were differentiated according to the part of the spine affected, including the disc. The most frequent pattern was the posterior arch (Fig. 1), followed by a pure vertebral body (Fig. 2).

The thoracic spine had the highest frequency of injury in our series. When we analyzed the associated lesions (Fig.
Table 3. Spinal injury pattern

\begin{tabular}{lc} 
Pattern & Frequency (\%) \\
\hline Posterior arch & $17(34.69)$ \\
\hline Vertebral body & $12(24.49)$ \\
\hline Vertebral body+unilateral pedicle & $6(12.24)$ \\
\hline Disc & $4(8.16)$ \\
\hline Both pedicles+posterior arch & $3(6.12)$ \\
\hline Unilateral pedicle & $3(6.12)$ \\
\hline Unilateral pedicle+posterior arch & $2(4.08)$ \\
Lateral mass & $1(2.04)$ \\
\hline Vertebral body+posterior arch & $1(2.04)$
\end{tabular}

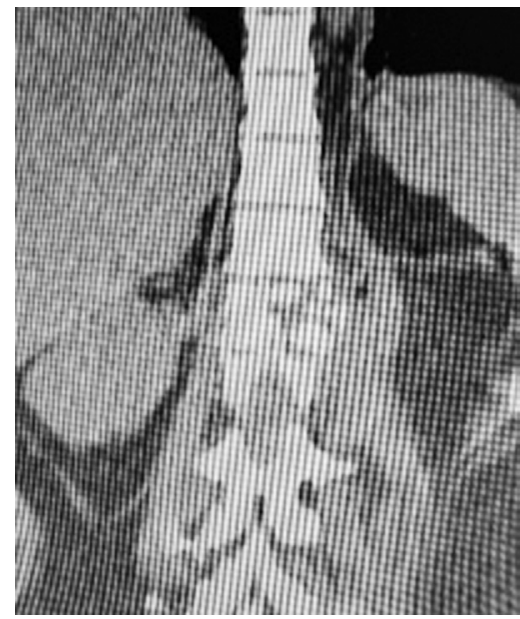

Fig. 2. Vertebral body pattern: Burst-type vertebral body fracture.

3 ), we found that the most frequently associated lesion was SCI (88\%), followed by hemopneumothorax (75\%), lung lesions (28\%), and hepatic lesions (16\%). The lumbar spine had the second highest frequency, with SCI being the most frequent (50\%), followed by lower GI tract and dural tear at $29 \%$ each; other lesions are shown in Fig. 4. 


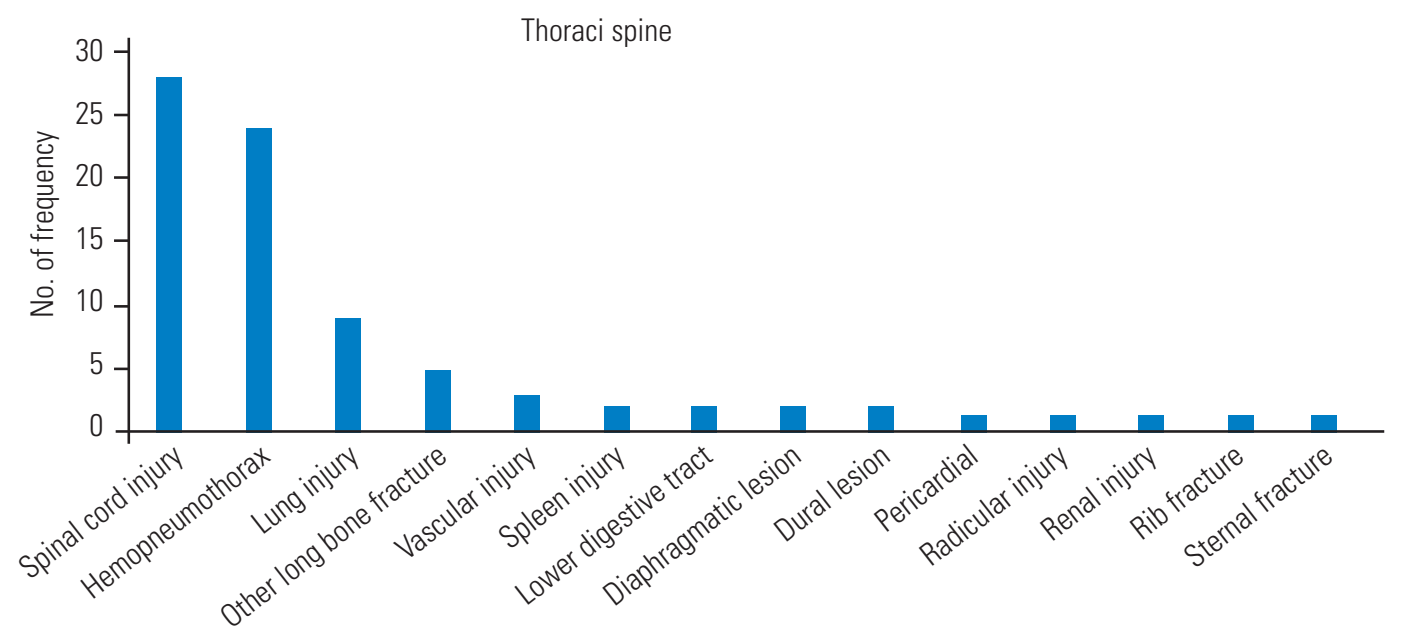

Fig. 3. Frequency of associated lesions in the thoracic segment

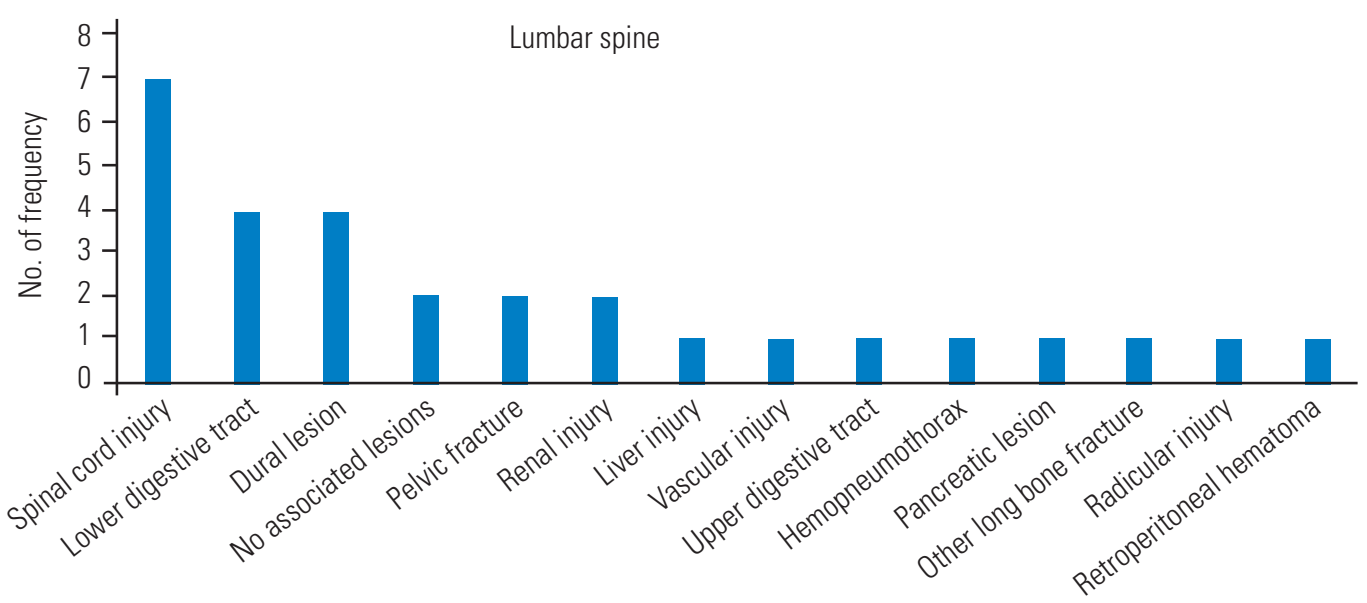

Fig. 4. Frequency of associated lesions in the lumbar segment.

When we compared the spinal injury pattern with SCI, we found that all patients with posterior arch involvement with one or both pedicles and $86 \%$ of patients with vertebral body involvement and unilateral pedicle had an SCI, as shown in Table 4. When we considered the presence of pedicle fracture, with or without vertebral body or posterior arch involvement, $86 \%$ had an SCI, resulting in an odds ratio of 3.64 (95\% confidence interval, 0.715-18.50) for severe SCI, with 11 Frankel A and 2 Frankel B grade injuries. The distribution of the spinal injury pattern related to SCI severity is shown in Table 5. Regarding patients with a vertebral body fracture and SCI as classified by spinal segment, eight injuries were in the thoracic spine and two were in the upper cervical spine, all of which had a Frankel A grade; the other two patients had injuries in the lumbar spine, of which one had a Frankel A and the other a Frankel B grade.
In the upper cervical spine case (C2), a mandibular fracture along with a retrogastric hematoma was reported, and the lateral mass was injured. In the subaxial spine cases, a vascular injury was reported in three patients, one root lesion, three complete SCI, one with partial SCI and two cases had no associated lesion.

We evaluated the relationship between the spinal injury pattern and associated lesions. We found that, of the patients with no associated injury (four cases), one had an injured disc and three had a posterior arch fracture. The bone fracture pattern with the most associated lesions was the vertebral body, with a total of 19 lesions, followed by the posterior arch, with five lesions, as shown in Table 6.

Of the hemopneumothorax cases, eight involved the posterior arch; six, the vertebral body; five, the vertebral body with unilateral pedicle; two, both pedicles and the posterior arch; two, the unilateral pedicle; one, the disc; 
Table 4. Spinal cord injury related to type of spinal injury

\begin{tabular}{|c|c|c|c|c|}
\hline \multirow{2}{*}{ Spinal bone pattern } & \multicolumn{4}{|c|}{ Spinal cord injury } \\
\hline & Total & No & Yes & $\%$ \\
\hline Both pedicles+posterior arch & 3 & 0 & 3 & 100 \\
\hline Unilateral pedicle+posterior arch & 2 & 0 & 2 & 100 \\
\hline Vertebral body+unilateral pedicle & 7 & 1 & 6 & 86 \\
\hline Vertebral body & 15 & 3 & 12 & 80 \\
\hline Posterior arch & 18 & 6 & 12 & 67 \\
\hline Unilateral pedicle & 3 & 1 & 2 & 67 \\
\hline Disc & 4 & 3 & 1 & 25 \\
\hline Lateral mass & 2 & 2 & 0 & 0 \\
\hline Total & 54 & 16 & 38 & 70 \\
\hline
\end{tabular}

Table 5. Severity of spinal cord injury distribution related to spinal injury pattern

\begin{tabular}{|c|c|c|c|c|c|c|}
\hline \multirow{2}{*}{ Spinal injury pattern } & \multicolumn{5}{|c|}{ Severity of spinal cord injury } & \multirow{2}{*}{ Total } \\
\hline & Frankel A & Frankel B & Frankel C & Frankel D & Frankel E & \\
\hline Both pedicles+posterior arch & 1 & 2 & 0 & 0 & 0 & 3 \\
\hline Disc & 1 & 0 & 0 & 0 & 3 & 4 \\
\hline Lateral mass & 0 & 0 & 0 & 0 & 2 & 2 \\
\hline Posterior arch & 10 & 0 & 1 & 2 & 5 & 18 \\
\hline Unilateral pedicle & 2 & 0 & 0 & 0 & 1 & 3 \\
\hline Unilateral pedicle+posterior arch & 2 & 0 & 0 & 0 & 0 & 2 \\
\hline Vertebral body & 11 & 1 & 0 & 0 & 3 & 15 \\
\hline Vertebral body+unilateral pedicle & 6 & 0 & 0 & 0 & 1 & 7 \\
\hline Total & 33 & 3 & 1 & 2 & 15 & 54 \\
\hline
\end{tabular}

Table 6. Associated lesion frequency according to spinal injury pattern

\begin{tabular}{|c|c|c|c|c|c|c|c|c|}
\hline \multirow{2}{*}{$\begin{array}{l}\text { Associated } \\
\text { lesion }\end{array}$} & \multicolumn{8}{|c|}{ Spinal injury pattern } \\
\hline & $\begin{array}{l}\text { Posterior } \\
\text { arch }\end{array}$ & $\begin{array}{l}\text { Vertebral } \\
\text { body }\end{array}$ & $\begin{array}{c}\text { Vertebral } \\
\text { body+unilateral pedicle }\end{array}$ & Disc & $\begin{array}{l}\text { Both pedicles and } \\
\text { posterior arch }\end{array}$ & $\begin{array}{l}\text { Unilateral } \\
\text { pedicle }\end{array}$ & $\begin{array}{l}\text { Unilateral } \\
\text { pedicle }\end{array}$ & $\begin{array}{l}\text { Lateral } \\
\text { mass }\end{array}$ \\
\hline Lung & 2 & 4 & 1 & & & 2 & & \\
\hline Hepatic & & 2 & & 1 & & & & \\
\hline Vascular & 1 & 4 & & & & & & 1 \\
\hline Spleen & 1 & & & 1 & & & & \\
\hline Lower Gl tract & 1 & 3 & 1 & & 1 & & 1 & \\
\hline Diaphragmatic & & 1 & 1 & 1 & & & & \\
\hline Pericardial & & & & & & 1 & & \\
\hline Renal & & 2 & & & 1 & & & \\
\hline Rib fracture & & 1 & & & & & & \\
\hline Sternum fracture & & 1 & & & & & & \\
\hline Upper Gl tract & & 1 & & & & & & \\
\hline Total & 5 & 19 & 3 & 3 & 2 & 3 & 1 & 1 \\
\hline
\end{tabular}

Gl, gastrointestinal. 
Table 7. Instrumented cases according to bony pattern

\begin{tabular}{lc} 
Spinal injury pattern & Frequency \\
Vertebral body & 5 \\
Posterior arch & 2 \\
Unilateral pedicle+vertebral body & 1 \\
Unilateral pedicle+posterior arch & 1 \\
Total & 9 \\
\hline
\end{tabular}

Table 8. Instrumented and decompression cases according to bony pattern

\begin{tabular}{lc} 
Spinal injury pattern & Frequency \\
Vertebral body & 3 \\
\hline Posterior arch & 1 \\
Both pedicles+posterior arch & 1 \\
Total & 5 \\
\hline
\end{tabular}

and one, the unilateral pedicle with the posterior arch. Six patients had dural injury, of whom the vertebral body was involved in two patients; the rest had an involvement of the posterior arch with pedicles, posterior arch with unilateral pedicle, or unilateral pedicle with posterior arch. For radicular injuries, the posterior arch was involved in three cases and the unilateral pedicle in one case.

Despite the severity of the associated lesions and the SCI, 78\% (38 patients) did not require intensive care unit (ICU) treatment. The mean length of stay in the ICU was $10.5 \pm 6.9$ days, with a range of $2-21$ days; $50 \%$ of the ICU cases lasted 2-4 days.

We found that 39 patients were treated conservatively, whereas 15 were treated surgically. Among the spinal procedures performed, nine were instrumented (Table 7), five instrumented with decompression (Table 8), and one with decompression only, which was a case of unilateral pedicle fracture. Of the surgical group, seven cases had the projectile removed because it was lodged in the spinal canal, and six (85\%) presented with a dural lesion. The decision for instrumentation was made according to spinal instability, considering the bone pattern, in which the burst fracture of the vertebral body was the main reason for instrumentation. Patients with bone fragments or a lodged bullet required decompression as well as instrumentation, primarily due to the instability after decompression. Only one patient had a lodged bullet with no instability criteria, and no instability was found after decompression.

\section{Discussion}

Patient distribution by age and sex is similar to that reported in the literature, with a high incidence in the young adult population, although there were cases of patients aged 9 and 75 years.

Kaim Khani et al. [2] studied patients with gunshot wounds in general and did not report a spinal injury pattern; some were high-energy injuries, and they did not focus on a particular segment. Unlike the study by Bumpass et al. [6], we describe a simpler pattern. We considered the posterior arch as a unit, given that spinal stability could be more seriously compromised by it than by its individual parts. We used the Magerl/AO fracture classification instead of the Denis three-column model when determining the stability of the vertebral body. We also considered subdividing the cervical segment into the upper and subaxial due to their differences in anatomy and function and in relation to important structures such as the skull, vertebral artery, and mandibular bone.

Compared with the study by Jakoi et al. [5], we had a higher incidence of lumbar gunshot wounds than of cervical gunshot wounds, even if both the upper and subaxial were considered together.

Chittiboina et al. [7] observed that the trajectory of the missile affected the pattern of the spinal gunshot wound in a predictable manner, in which patients who were shot from behind had a posterior arch fracture. Patients who were shot from the front had a vertebral fracture, and those who were shot from the side had pedicle fractures. We also observed this result when relating the fracture pattern to the associated lesions. As with their series, we noted that in the cases in which the pedicles were involved, there was a very high chance of having a severe or complete SCI. We also found that the thoracic spine was more frequently involved in SCI, possibly due to the transfer of energy to the spinal cord, whereas in the lumbar spine the vertebrae are larger and could absorb this energy better, also the spinal cord.

As in the study by Waters and Sie [3], we noted that spinal stability was not compromised if one of the pedicles or facet joints remained intact. Therefore, spinal instability was mainly secondary to vertebral body burst-type fractures (eight cases out of 15). Considering the high risk of evolving into a kyphotic deformity, this type of fracture was the main reason for instrumentation. The second indication for surgery was lodging of the projectile within 

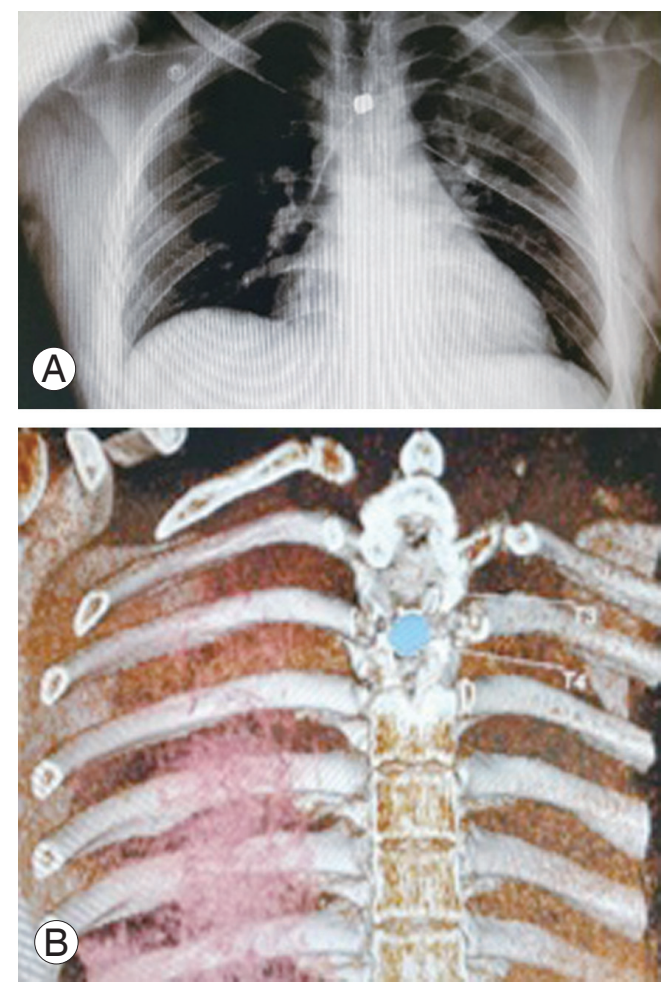

Fig. 5. (A) Bullet lodged in spinal canal: simple X-ray with bullet lodged in upper thoracic level with a pleural tube. (B) Bullet lodged in spinal canal: computed tomography reconstruction of bullet lodged between T3 and T4.

the spinal canal (Fig. 5). In these cases, we recommend the extraction of the projectile due to the high incidence of dural lesions, as found in our series, that can increase the risk of metal (lead) exposure to the central nervous system. In addition, in patients with debris or bone fragments lodged in the spinal canal, treatment required decompression with or without instrumentation, depending on spinal stability.

Concerning the associated lesions, we found that lower GI tract perforation had a great impact, if not the greatest impact of all associated lesions, on the prognosis and treatment. In our experience, this type of injury delays and can even contraindicate surgery due to the high probability of surgical site infection. One of the patients with a lower GI tract lesion was treated and considered for instrumentation, but once in surgery an abscess was found; thus, the patient was treated with debridement and was administered a specific antibiotic treatment based on the culture report.

\section{Conclusions}

We found that the impact of the bullet to the spine created 8 different spinal injury patterns in our series. Based on their frequency, we found two main patterns (the vertebral body and posterior arch) with the pedicles as a modifier because they represent a higher risk for complete or severe SCI. These patterns are usually stable in nature, unless it is a burst fracture of the vertebral body. Vertebral body fractures have a higher frequency of associated lesions independent of the spinal segment due to their anatomical location. The highest incidence of SCI was found in the thoracic spine, with a similar distribution between anterior and posterior spinal patterns. The main indications for surgery were instability, mostly secondary to vertebral body burst fracture, and bullet removal when it was lodged in the spinal canal. Associated injuries influenced the clinical treatment decision, given that they had to be treated with greater urgency than the spinal injury itself. In addition, in some cases, associated injuries can aggravate the patient's overall health and delay surgery or even contraindicate surgical treatment when necessary. Therefore, the treatment decision remains complex and challenging.

\section{Conflict of Interest}

No potential conflict of interest relevant to this article was reported.

\section{References}

1. Hernandez-Tellez IE, Montelongo-Mercado E, Arreola-Bastidas JJ, Garcia-Valadez LR, SanchezArellano JL, Hernandez-Gomez N. Epidemiology of injuries to the spine by gun fire. Rev Sanid Milit Mex 2015;69:265-74.

2. Kaim Khani GM, Humail SM, Hafeez K, Ahmed N. Pattern of bony injuries among civilian gunshot victims at tertiary care hospital in Karachi, Pakistan. Chin J Traumatol 2015;18:161-3.

3. Waters RL, Sie IH. Spinal cord injuries from gunshot wounds to the spine. Clin Orthop Relat Res 2003;(408):120-5.

4. Sidhu GS, Ghag A, Prokuski V, Vaccaro AR, Radcliff KE. Civilian gunshot injuries of the spinal cord: a 
systematic review of the current literature. Clin Orthop Relat Res 2013;471:3945-55.

5. Jakoi A, Iorio J, Howell R, Zampini JM. Gunshot injuries of the spine. Spine J 2015;15:2077-85.

6. Bumpass DB, Buchowski JM, Park A, et al. An update on civilian spinal gunshot wounds: treatment, neurological recovery, and complications. Spine (Phila $\mathrm{Pa}$ 1976) 2015;40:450-61.

7. Chittiboina P, Banerjee AD, Zhang S, Caldito G, Nanda A, Willis BK. How bullet trajectory affects outcomes of civilian gunshot injury to the spine. J Clin Neurosci 2011;18:1630-3.

8. Jimenez-Avila JM, Cobar-Bustamante AE, CahuequeLemus MA, Gutierrez-Roman EA, Guzman-Pantoja JE. Firearm injuries in the spine: effective strategy for diagnosis and treatment. Coluna/Columna 2015;14:240-3.

9. Tejeda-Barreras M. Gun injuries to the spine. Orthotips 2011;7:155-61.

10. Rentfrow B, Vaidya R, Elia C, Sethi A. Lead toxicity and management of gunshot wounds in the lumbar spine. Eur Spine J 2013;22:2353-7.
11. Kahraman S, Gonul E, Kayali H, et al. Retrospective analysis of spinal missile injuries. Neurosurg Rev 2004;27:42-5.

12. Zura RD, Bosse MJ. Current treatment of gunshot wounds to the hip and pelvis. Clin Orthop Relat Res 2003;(408):110-4.

13. De Barros Filho TE, Cristante AF, Marcon RM, Ono A, Bilhar R. Gunshot injuries in the spine. Spinal Cord 2014;52:504-10.

14. Bhatoe HS, Singh P. Missile injuries of the spine. Neurol India 2003;51:507-11.

15. Kumar A, Pandey PN, Ghani A, Jaiswal G. Penetrating spinal injuries and their management. J Craniovertebr Junction Spine 2011;2:57-61.

16. Jaiswal M, Mittal RS. Concept of gunshot wound spine. Asian Spine J 2013;7:359-64.

17. Trahan J, Serban D, Tender GC. Gunshot wounds to the spine in post-Katrina New Orleans. Injury 2013;44:1601-6. 